\title{
Namensänderungen strafrechtlich Verurteilter nach identifizierender Medienberichterstattung
}

In den letzten zehn Jahren wurden in der Schweiz mindestens 18 Gesuche auf Namensänderungen von strafrechtlich verurteilten Personen eingereicht, oftmals begründet mit einer identifizierenden medialen Berichterstattung. Von den Kantonen wurden diese mehrheitlich abgelehnt. In diesem Aufsatz wird aufgezeigt, dass diese Praxis zu restriktiv ist, da bei identifizierender Berichterstattung und damit einhergehender Stigmatisierung einer Person als kriminell die Namensänderung zur Beseitigung der resultierenden Nachteile offenstehen muss. Wir argumentieren, dass die Ablegung dieses Stigmas einen achtenswerten Grund darstellt und zudem eine allfällige Missbräuchlichkeit der Namensänderung einfach verhindert werden kann. Weiter gehen wir auf den Zeitpunkt der Änderung ein und sprechen uns dafür aus, diesen bereits während des Vollzugs einer allfälligen Inhaftierung anzusetzen.

I. Einleitung

II. Rechtliche Voraussetzungen der Namensänderung

III. Praxis der kantonalen Behörden

IV. Zulässigkeit der Namensänderungen Inhaftierter und Strafentlassener

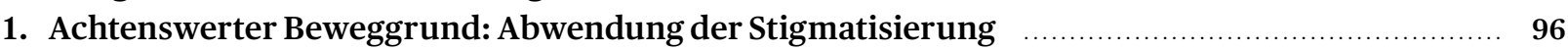

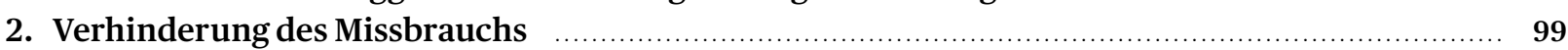

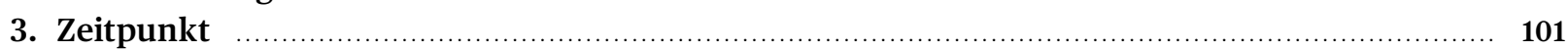

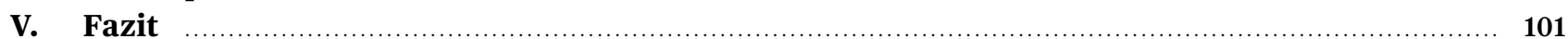

Zitiervorschlag: BENJAMIN STÜCKELBERGER/ EVIN YESILÖZ / DAMIAN CAVALLARO, Namensänderungen strafrechtlich Verurteilter nach identifizierender Medienberichterstattung, sui generis 2021, S. 93

Benjamin Stückelberger, BLaw, Wissenschaftlicher Hilfsassistent am Institut für Strafrecht und Kriminologie der Universität Bern (benjamin.stueckelberger@krim.unibe.ch).Evin Yesilöz, MAS in Transitional Justice and Human Rights, Wissenschaftliche Hilfsassistentin am Institut für Völkerrecht und ausländisches Verfassungsrecht der Universität Zürich (evin.yesiloez@rwi.uzh.ch).Damian Cavallaro, MLaw, Wissenschaftlicher Assistent am Institut für Strafrecht und Kriminologie der Universität Bern (damian.cavallaro@

krim.unibe.ch). Ein grosser Dank geht insbesondere an Prof. Dr. Jonas Weber sowie die anonyme Gutachtensperson für wertvolle Hinweise und Kommentare.

URL: sui-generis.ch/172

DOI: https://doi.org/10.21257/sg.172

Dieses Werk ist lizenziert unter einer Creative Commons Namensnennung - Weitergabe unter gleichen Bedingungen 4.0 International Lizenz. 


\section{Einleitung}

1 Im April 2020 strahlte das Radio SRF einen Beitrag mit dem Titel «Heute vor 34Jahren: Amoklauf von Günther Tschanun» aus. 1986 erschoss der damalige Chef der Zürcher Baupolizei nach Differenzen am Arbeitsplatz vier Mitarbeiter und wurde zu 20Jahren Haft verurteilt. Zwölf Jahre später wurde er aus der Haft entlassen. Über seinen heutigen Aufenthaltsort, oder ob er gar noch lebt, kann allerdings nur spekuliert werden - denn nach dem Strafvollzug liess Günther Tschanun seinen Namen ändern. ${ }^{1}$

2 Namensänderungen wurden von Lehre und Rechtsprechung bisher vor allem im familienrechtlichen Kontext oder bzgl. verunstaltender Namen behandelt, ${ }^{2}$ Namensänderungen von strafrechtlich Verurteilten nach identifizierenden Medienberichten hingegen gaben bisher keinen Anlass zur Diskussion. ${ }^{3}$ U.E. wird dieses Thema jedoch an Relevanz gewinnen, insbesondere angesichts des Wandels zur Informationsgesellschaft und stetig wachsenden digitalen Archiven. Letztere ermöglichen insbesondere in Kombination mit Suchmaschinen - die Konfrontation ehemaliger Straftäterinnen und Straftäter mit begangenen Taten auch Jahre nach ihrer Verurteilung, was einen grossen Einfluss auf den Resozialisierungsprozess und die positive Spezialprävention hat. ${ }^{4}$

3 Dieser Aufsatz soll deshalb die Thematik wissenschaftlich aufarbeiten und die Forschungsfragen beantworten, inwiefern Namensänderungsgesuche von strafrechtlich verurteilten Personen zugelassen werden sollen und ob die momentane kantonale Praxis dem entspricht. In einem ersten Schritt (II.) sollen die rechtlichen Voraussetzungen von Namensänderungen nach Art. 30 Abs. 12GB5 im Generellen dargestellt werden. Anschliessend (III.) wird die heutige Praxis der Kantone zur Namensänderung strafrechtlich Verurteilter aufgezeigt, die mittels einer Umfrage bei den kantonalen Behörden erhoben worden ist. Daraufhin (IV.) wird die Zulässigkeit der Namensänderung von verurteilten Personen besprochen und dabei auch auf die zuvor dargestellte kantonale Praxis Bezug genommen. Zuerst soll dabei die Stigmatisie-

1 Radio SRF vom 16. April 2020 (Heute vor 34 Jahren: Amoklauf von Günther Tschanun).

2 ROLAND BÜHLER, in: Geiser/Fountoulakis (Hrsg.), Zivilgesetzbuch I, Basler Kommentar, 6. Aufl., Basel 2018, Art. 30 N 8 ff. (zit. BSK ZGB I-BEARBEITERIN).

3 Anders in Deutschland, wo die Thematik sogar auf Verordnungsstufe geregelt ist, siehe Nr.39 Abs. 1 Allgemeine Verwaltungsvorschrift zum Gesetz über die Änderung von Familiennamen und Vornamen (NamÄndVwV) vom 11. August 1980.

4 Vgl. SARAH E. LAGESON / SHADD MARUNA, Digital degradation: Stig ma management in the internet age, Punishment \& Society 2018, S. 125 .

5 Schweizerisches Zivilgesetzbuch vom 10. Dezember 1907 (ZGB; SR 210). rung durch eine identifizierende Medienberichterstattung als achtenswerter Grund für eine Namensänderung besprochen werden, anschliessend soll eine mögliche Missbrauchsgefahr eingehend erläutert und zum Schluss auf den Zeitpunkt des Namenswechsels eingegangen werden, bevor wir den Aufsatz mit unserem Fazit (V.) abschliessen.

Es gilt anzumerken, dass sich dieser Aufsatz mit der Na- 4 mensänderung strafrechtlich Verurteilter befasst, also Personen, die in ihrem Leben für eine Straftat verurteilt und deswegen eine bedingte oder unbedingte Strafe resp. eine Massnahme angeordnet wurde. Selbstverständlich kann eine Berichterstattung aber auch bei einem formellen Freispruch eine stigmatisierende Wirkung entfalten. Somit können die gemachten Ausführungen, vorbehältlich der mit einem Freispruch verbundenen Möglichkeiten - etwa nach Art. 28 ff. ZGB oder Art. 68 Abs. 2 StGB $^{6}$ deren Erörterung den Rahmen dieses Aufsatzes sprengen würde, u.U auch für diese Personen Anwendung finden.

Auch ist darauf hinzuweisen, dass die eherechtliche Na- 5 mensänderung nicht besprochen wird, da diese für die vorliegende Thematik keine besonderen Probleme bereitet. ${ }^{7}$ Ferner sei angesprochen, dass bei ehemaligen Straftäterinnen und Straftätern selbstverständlich auch andere Gründe für eine Namensänderung bestehen können als Stigmatisierung aufgrund einer identifizierender Medienberichterstattung. Da sich hierbei u.E. - ausser einer allfälligen Missbrauchsgefahr, welche hier losgelöst von den Gründen für die Namensänderung diskutiert wird - jedoch kaum Besonderheiten ergeben, sei dafür auf die entsprechende Literatur und Rechtsprechung verwiesen. ${ }^{8}$

\section{Rechtliche Voraussetzungen der Namensänderung}

Die Änderung des Namens einer natürlichen Person 6 richtet sich nach Art. 30 Abs.1 ZGB. Dieser Artikel statuiert den Grundsatz der Unabänderlichkeit des Namens, ermöglicht jedoch dessen Änderung beim Vorliegen «achtenswerter Gründe». ${ }^{9}$ Diese Formulierung ist seit dem 1.Januar 2013 in Kraft und ersetzte den Terminus «wichtige Gründe». Das Bundesgericht hielt dazu fest, dass mit der Neufassung des Artikels beabsichtigt wurde,

6 Schweizerisches Strafgesetzbuch vom 21. Dezember 1937 (StGB; SR311.0).

7 Zudem wurde uns lediglich ein solcher Fall von den kantonalen Behörden mitgeteilt.

8 Vgl. statt vieler: THOMAS GEISER, Das neue Namensrecht und die Kindes- und Erwachsenenschutzbehörde, ZKE 2012, S. $353 \mathrm{ff}$.

9 BSK ZGB I-BÜHLER, Art. 30 N1, REGINA E. AEBI-MÜLLER, Das neue Familienrecht - eine erste Übersicht, SJZ 2012, S. 455. 
die Hürden für eine Namensänderung zu senken, ohne jedoch jeder Person die Möglichkeit zum beliebigen Namenswechsel zu geben, da der Name einer Person grundsätzlich unabänderlich bleiben sollte. ${ }^{10}$

7 Achtenswert sind die Beweggründe bereits dann, wenn sie «nicht geradezu belanglos, sondern einsichtig erscheinen». ${ }^{11}$ Als Grundlage für die Namensänderung können objektive, in der Natur des Namens liegende, sowie subjektive, nur in Verbindung mit der spezifischen Person Geltung erlangende Unannehmlichkeiten herangezogen werden, sofern diese Gründe einen gewissen Schweregrad erreichen und nicht völlig zwecklos sind. ${ }^{2}$ In Lehre und Rechtsprechung dafür aufgeführte Beispiele betreffen bspw. Pseudonyme, welche zu amtlichen Namen gemacht wurden, ${ }^{13}$ die Hervorhebung einer Religionszugehörigkeit oder die Verdeckung ausländischer Herkunft. ${ }^{14}$

8 Ob ein achtenswerter Grund vorliegt, muss unter Berücksichtigung aller sachlich wesentlichen Umstände des Einzelfalls nach Recht und Billigkeit beantwortet werden. ${ }^{15}$ Weiter ist auch zu beachten, dass die achtenswerten Gründe und die angestrebte Namensänderung «nicht rechtswidrig, missbräuchlich oder sittenwidrig» 16 sein dürfen.

9 Der Vollständigkeit halber ist zu bemerken, dass bei einer Namensänderung der neue Name gesetzeskonform sein muss und Rechte Dritter nicht verletzen darf. ${ }^{17} \mathrm{Da}$ sich hierbei jedoch keine besonderen Problematiken für strafrechtlich Verurteilte ergeben, wird an dieser Stelle nicht näher darauf eingegangen.

\section{Praxis der kantonalen Behörden}

10 den Fragebögen an sämtliche kantonal zuständige Behörden gesandt (26), deren 25 (Rücklaufquote: $96 \%$ ) antworteten darauf; davon erhielten wir von 20 Behörden

10 BGE140 III 577 E. 3.3.3 S. 581; BGE145 III 49 E.3.2 S. 51ff.; wobei in der Auslegung wohl zu beachten ist, dass eine Namensänderung das Recht auf Privatleben nach Art. 8 EMRK, resp. Art. 13 Abs. 1 BV berührt, vgl. Urteil des EGMR12209/10 vom15. September 2015(Macalin Moxamed Sed Dahier gegen die Schweiz), \$21f.

11 BSK ZGBI-BÜHLER, Art. 30 N 9; vgl. auch AEBI-MÜLLER (Fn.9), S. 456f.; MELANIE BÜRKI / STEPHANIE HRUBESCH-MILAUER, Rechtsprechungspanorama Personenrecht und Einleitungsartikel, AJP 2019, S. $1045 \mathrm{f}$.

12 BGE145III 49 E.3.2 S. 52 ff.; BSK ZGB I-BÜHLER, Art. 30 N 5; HEINZ HAUSHEER / REGINA E. AEBI-MÜLLER, Das Personenrecht des Schweizerischen Zivilgesetzbuches, 4. Aufl., Bern 2016, N16, 39.

13 Urteil des Bundesgerichts 5A_730/2017 vom 22.Januar 2018 E.3.2.

14 BSKZGB I-BÜHLER, Art. 30 N 9.

15 BGE132 III 497 E. 2; BSK ZGB I-BÜHLER, Art. 30 N 5 f.

16 GEISER (Fn. 8), S. 371; BSK ZGB I-BÜHLER, Art.30 N9.

17 BGE145III 49 E.3.2 S. 52 ff. eine inhaltliche Auskunft (77\%). ${ }^{18}$ Der Fragebogen ersuchte um Bereitstellung der Daten der Anzahl von Anträgen um Namensänderungen über einen Zeitraum von zehn Jahren ${ }^{19}$ von strafrechtlich verurteilten Personen, der Gutheissungs- resp. Ablehnungsquote und die Gründe für die jeweilige Entscheidung. Zudem wurde der Zeitpunkt des Antrags im allfälligen Vollzugsverlauf einer Strafe erfragt.

Aus den Antworten ging hervor, dass im Zeitraum min- 11 destens 18 Gesuche oder informelle Anfragen auf Namensänderung von strafrechtlich Verurteilten gestellt wurden, woraus zu schliessen ist, dass der Wunsch nach Namensänderung strafrechtlich Verurteilter zwar kein tagtägliches Geschäft ist, sich die Problematik aber auch in der Schweiz wiederholt stellt.

Bei sieben Anfragen wurden die Gesuchsgründe für die 12 Namensänderung durch die kantonalen Behörden nicht bekannt gegeben, in weiteren drei Fällen standen sie nicht in unmittelbarem Zusammenhang mit der Straftat. In acht Fällen wurden jedoch von den Gesuchstellenden Namensnennungen in den Medien - und damit verbunden die leichte Auffindbarkeit im Internet sowie die damit verknüpften Nachteile nach der Haftentlassung - als Grund für die gewünschte Namensänderung vorgebracht. Der Zweck des neuen Namens wurde in diesen Fällen darin gesehen, ein neues Leben beginnen zu können. Bei letztgenannter Argumentation erscheint die Entscheidpraxis - ohne die Umstände der Einzelfälle im Detail zu kennen - überwiegend ablehnend: Uns ist lediglich ein in dieser Art begründetes und anschliessend gutgeheissenes Gesuch bekannt. Sämtliche weiteren vergleichbaren Gesuche wurden abgelehnt. Dabei lassen sich vier Argumentationsstränge erkennen: Erstens wurde vorgebracht, dass das Interesse der Öffentlichkeit an der verurteilten Person kein achtenswerter Grund für eine Namensänderung darstelle, resp. die grundsätzliche Unabänderlichkeit des Namens höher zu gewichten sei (vgl. IV.1). Zweitens wurde argumentiert, dass die Namensänderung kein geeignetes Mittel zur Abwendung des Interesses der Öffentlichkeit sei, da der neue Name vor den Medien i.d.R nicht lange standhalte und potenzielle Arbeitgebende dennoch von den begangenen Straftaten Kenntnis erhalten würden (vgl. ebenfalls IV.1).

18 Dabei erhielten wir Antworten von allen Kantonen ausser Wallis; die Kantone Appenzell Innerrhoden, Genf, Solothurn, St. Gallen und Zürich konnten mangels Statistikführung jedoch keine Daten zur Verfügung stellen. Zu beachten ist, dass zahlreichen weiteren Kantonen lediglich Daten über einen kürzeren Zeitraum zur Verfügung standen.

19 Versandt wurden die Fragebögen am 30. Mai 2020, womit sich der erfragte Zeitraum vom 1. Juni 2010 bis zu diesem Datum erstreckt. Anzumerken ist, dass das Namensänderungsrecht auf den 1.Januar 2013 revidiert wurde und aus den bereitgestellten Daten nicht ersichtlich ist, ob die Entscheide vor oder nach deren Revision getroffen wurden. 
Drittens wurde ein Gesuch abgelehnt, da es sehr früh im Haftverlauf gestellt wurde und gemäss der zuständigen Namensänderungsbehörde zu diesem Zeitpunkt die Resozialisierung nicht im Zentrum stünde (vgl. IV.3). Zuletzt wurde in mehreren Entscheiden auf eine Missbräuchlichkeit hingewiesen, welche durch Verschaffung einer «weissen Weste» resp. der durch die Namensänderung nicht mehr nachvollziehbaren kriminellen Vergangenheit gegeben sei (vgl. IV.2). den Ergebnis zwar oftmals überein, bei den für die Gesuchabweisung behändigten Argumenten besteht indes Heterogenität.

\section{Zulässigkeit der Namens- änderungen Inhaftierter und Strafentlassener}

\section{Achtenswerter Beweggrund: Abwendung der Stigmatisierung}

14 Die Funktionen eines Namens sind mannigfaltig; er gewährleistet Identifikation und Unterscheidung von anderen, ist aber auch ein gewichtiger Teil der eigenen Identität. ${ }^{20}$ Haben strafrechtlich Verurteilte den Antrag auf Namensänderung nun mit einem gewünschten Neuanfang im Leben begründet, kann angenommen werden, dass der bis anhin getragene Name mit der begangenen Straftat verknüpft ist und ihm somit das Stigma kriminell anhaftet. ${ }^{21}$ Im Folgenden wird ergründet, worin dieses Stigma besteht, welche Auswirkungen es hat, in welcher Weise es eine Publizitätswirkung entfaltet und wann es als achtenswerter Grund für eine Namensänderung herangezogen werden kann.

\section{a) Das Stigma kriminell und dessen Auswirkungen}

15 Als Stigma wird die Herabwürdigung einer Person verstanden, «indem sie wegen eines Merkmals oder einer Eigenschaft als ‘anders` und weniger fähig, weniger intelligent, weniger vertrauenswürdig oder weniger verträglich angesehen und behandelt wird».22 Ein stigmatisierendes Merkmal kann unterschiedlicher Art sein. Goffmann unterscheidet drei Kategorien von Faktoren, welche zur Stigmatisierung führen können: die der körperlichen (wie Geschlecht oder körperliche Behinderungen), der intergenerationalen (wie Migrationshintergrund oder sozialer Status) und der verhaltensbezogenen (wie Kriminalität und psychische Erkrankungen)

20 BIRGIT VÖLLM / LIZ JAMIESON / PAMELA J. TAYLOR, What's in a name? Reasons for changing names among english high security hospital patients, The Journal of Forensic Psychiatry \& Psychology 2006, S. 37.

21 Vgl. dazu vÖLlM/JAMIESON/TAYLOR (Fn. 20), S. 38, 45, 49.

22 PHILIPP SCHULTE, Kontrolle und Delinquenz, Münster 2019, S. 25.
Faktoren. ${ }^{23}$ Insbesondere die Zuschreibung des Merkmals kriminell ist dabei einer der «schärfsten institutionellen Ausgrenzungsmechanismen moderner Gesellschaften». ${ }^{44}$ Diese Etikettierung führt zu einer Änderung der Wahrnehmung Dritter der betroffenen Person - so wird stärker angenommen, dass diese «unehrlich, gewalttätig, respektlos, unsozial» sei ${ }^{25}$ und sich generell normwidriger verhalte. ${ }^{26}$ Becker beschreibt Letzteres als inneren Dialog des Gegenüber: «The question is raised: «What kind of person would break such an important rule?» And the answer is given: «One who is different from the rest of us, who cannot or will not act as a moral human being and therefore might break other important rules.»»27 Das Besondere am Labeling einer Person ist dabei, dass dies sogar dazu führen kann, dass Handlungen, welche bei anderen positiv wahrgenommen werden, bei ihr negativ bewertet werden, da die Zuschreibung als kriminell dominiert. ${ }^{28}$

Durch diese Effekte entstehen zahlreiche Auswirkungen 16 auf den Alltag betroffener Personen. Empirische Forschungsergebnisse zeigen, dass als Folge häufig Schwierigkeiten bei der Suche einer Arbeitsstelle und Wohnung bestehen. ${ }^{29}$ Zudem ist es oftmals schwierig, sich wieder in die Gesellschaft einzugliedern und in ihr zu partizipieren, ferner nimmt auch die Gefahr psychischer Erkrankungen zu. ${ }^{30}$ Ebenfalls kann diese Zuschreibung die Selbstidentifikation als «rechtschaffene» Person erschweren und damit verknüpft die Wahrscheinlichkeit erneuter Delinquenz steigern. ${ }^{31}$

\section{b) Publizität und das Stigma}

Voraussetzung für die beschriebenen Effekte ist die Pu- 17 blizität der Tat, ${ }^{32}$ d.h. die in Teilen der Bevölkerung vorhandene Kenntnis über die Begehung der Straftat einer bestimmten Person, anderweitig gar kein Stigma entstehen kann. Diese Publizität ist bis zu einem gewissen

23 ERVING GOFFMANN, Stigma: Notes on the Management of Spoiled Identity, New York 1963, S. 4.

24 SCHULTE (Fn. 22), S. 20.

25 SCHULTE (Fn. 22), S. 27.

26 VIKTOR PICKL, Stigmatisierung durch Strafverfahren und Strafvollzug, in: Haesler (Hrsg.), Stigmatisierung durch Strafverfahren und Strafvollzug, Diessenhofen 1981, S. 35.

27 HOWARD S. BECKER, Outsiders: Studies in the Sociology of Deviance, New York 1963, S. $33 \mathrm{f}$.

28 BECKER, (Fn. 27), S.33f.; SCHULTE (Fn. 22), S. 27.

29 SCOTT H. DECKER / NATALIE ORTIZ / CASSIA SPOHN / ERIC HEDBERG, Criminal stigma, race, and ethnicity: The consequences of imprisonment for employment, Journal of Criminal Justice 2015, S. 108ff.; DANYA E. KEENE / AMY B. SMOYER / KIM M. BLANKENSHIP, Stigma, hou sing and identity after prison, The Sociological Review 2018, S.799ff.

30 Vgl. KELLY E. MOORE / JEFFREY B. STUEWIG / JUNE P. TANGNEY, The Effect of Stigma on Criminal Offenders' Functioning: A Longitudi nal Mediational Model, Deviant Behavior 2016, S.196ff. m.w.H.

31 LAGESON/MARUNA (Fn. 4), S.115, 124 f.; SCHULTE (Fn. 22), S. $21 \mathrm{ff}$ 32 PICKL (Fn. 26), S. 40. 
Grad gesetzlich vorgesehen durch die Gerichtsöffentlichkeit, welche verfassungsrechtlich geschützt ${ }^{33}$ und durch Art. 69 Abs. 1 StPO ${ }^{34}$ auch ins Strafprozessrecht übertragen wurde. ${ }^{35}$

Mit der Gerichtsöffentlichkeit geht die Berichterstattung über die gerichtliche Tätigkeit einher, welche in einem demokratischen Rechtsstaat unbestrittenermassen von grosser gesellschaftlicher und politischer Bedeutung ist, ${ }^{36}$ denn erst durch die Justizberichterstattung wird eine wahrhaftige Gerichtsöffentlichkeit für ein breites Publikum hergestellt. Vom Bundesgericht wird ihr sowohl eine Brückenfunktion als auch eine Wächterrolle zugemessen. Insofern, als die Gerichtsberichterstattung den korrekten und gesetzmässigen Prozessablauf kontrolliert, dient sie auch der beschuldigten Person. ${ }^{37}$ Daneben kann sie indessen auch eine Stigmatisierung bewirken. Wird in der Gerichtsberichterstattung der Name der beschuldigten Person genannt oder diese anderweitig identifizierbar dargestellt, ${ }^{38}$ findet unweigerlich eine Verknüpfung von Straftat und Person statt. Dadurch kann eine beträchtliche stigmatisierende Publizitätswirkung entstehen, das Bundesgericht spricht gar von einer «Prangerwirkung». ${ }^{39}$ Dies geschieht unabhängig davon, ob es sich um eine gerechtfertigte oder eine widerrechtliche persönlichkeitsverletzende Berichterstattung handelt. ${ }^{40}$

19 Im Zeitalter des Internets erlangt diese Berichterstattung eine neue Tragweite: Durch die Digitalisierung der Medienarchive (z.B. Swissdox oder Factiva) sind identifizie-

33 Art. 30 Abs. 3 BV (Bundesverfassung der Schweizerischen Eidgenossenschaft vom 18. April 1999 [BV; SR 101]); Art. 6 Abs. 1 EMRK (Konvention zum Schutze der Menschenrechte und Grundfreiheiten, abgeschlossen in Rom am 4. November 1950 [EMRK; SR 0.101]); Art. 14 UNO Pakt II (Internationaler Pakt über bürgerliche und politische Rechte [UNO Pakt II; SR 0.103.2]).

34 Schweizerische Strafprozessordnung vom 5. Oktober 2007 (Strafprozessordnung, StPO; SR 312.0).

35 Wobei nach dem Bundesgericht die beschuldigte Person die mit einer öffentlichen Verhandlung verbundenen psychischen Belastungen und Konsequenzen grundsätzlich zu erdulden hat (Urteil des Bundesgerichts 1B_87/2018 vom 9. Mai 2018 E.3.2.4).

36 MATTHIAS MAHLMANN, Rechtsstaat, Demokratie, Öffentlichkeit, in: Kipfer/Kühler (Hrsg.), Justizberichterstattung in der direkten Demokratie, Stuttgart 2019, S. 32; siehe auch Urteil des Bundesgerichts 1B_87/2018 vom 9. Mai 2018 E.3.2.1.

37 BGE143I194 E.3.1 S.197ff.

38 Zu einer Identifizierung kann es beispielsweise durch die Veröffentlichung des Vornamens und des ersten Buchstabens des Nachnamens, dem Abdrucken von Bildern, gewissen Informationen über Beruf oder Wohnort oder durch eine unglückliche Kombination vorgenannter Dinge kommen.

39 BGE 109 II 353 E. 3 S. 356 ff.; BGE 119 Ia 99 E. 4b S. 105f.; BGE 120 IV 348 E.1aS.350f.

40 Deren Abgrenzung hier nicht im Vordergrund steht, dafür verweisen wir auf die einschlägige Literatur und Rechtsprechung, insbesondere MIRJAM TEITLER, Der rechtskräftig verurteilte Straftäter und seine Persönlichkeitsrechte im Spannungsfeld zwischen öffentlichem Informationsinteresse, Persönlichkeitsschutz und Kommerz, Zürich 2008; BGE127III 481; BGE129III 529. rende und stigmatisierende Berichte potenziell unbefristet für die breite Bevölkerung zugänglich. Während eine erneute Berichterstattung über verurteilte Personen ohne neues Ereignis (wie bspw. einen Rückfall) nach Abschluss des Verfahrens meist widerrechtlich ist, ${ }^{41}$ sieht das schweizerische Rechtssystem in Bezug auf die bestehende Berichterstattung jedoch kein Recht auf Vergessen vor, welches die Löschung persönlicher Datenspuren im Internet ermöglichen und dadurch den $\mathrm{Zu}$ gang für Drittpersonen einschränken würde. ${ }^{42}$ Bei widerrechtlicher identifizierender Berichterstattung stehen der betroffenen Personen allenfalls zum Rechtsschutz die Klagen von Art. 28a ZGB (i.V.m. Art. 15 DSG ${ }^{43}$ ) zur Verfügung. Allerdings muss hierbei gegen jedes veröffentlichende Organ einzeln vorgegangen werden, was insbesondere im Falle von andernorts hochgeladenen Kopien der Berichterstattung, bspw. auf privaten Blogs, beträchtliche Schwierigkeiten hervorrufen kann. War die identifizierende Berichterstattung im Zeitpunkt der Veröffentlichung hingegen gerechtfertigt, greift der Rechtsschutz des Art. 28a ZGB nicht. Auch der EGMR hat das Interesse an einer nachträglichen Anonymisierung von Medienartikeln zur Verhinderung weiterer Konfrontation mit den vergangenen Straftaten weniger starkgewichtet als die Medienfreiheit und das Interesse der Öffentlichkeit, Nachforschungen zu vergangenen Ereignissen tätigen zu können. ${ }^{44}$ In Bezug auf gerechtfertigte persönlichkeitsverletzende Berichterstattung ist somit die Möglichkeit der Löschung stark begrenzt und hängt stark von der Mitwirkung der Seitenbetreibenden ab. ${ }^{45}$ In diesem Fall, ebenso wie in Fällen rechtswidriger Berichterstattung, deren Beseitigung mit erheblichen Schwierigkeiten verbunden ist, bietet sich eine Namensänderung zur Abwendung des mit der Straftat verbundenen Stigmas an.

\section{c) Das Stigma kriminell als achtenswerter Grund zur Namensänderung}

Das Labeling einer Person als kriminell hat, wie beschrie- 20 ben, zahlreiche negative Konsequenzen, welche durch

41 BGE109II353 E. 4b S.360f.; TEITLER (Fn. 40), S. 84 ff.; hierfür spricht auch die Anonymisierung der Urteile.

42 ULRIKE I. HEINRICH / ROLF H. WEBER, Braucht die Schweiz ein Recht auf Vergessen im Internet?, in: Epiney/Diezig (Hrsg.), Schweizerisches Jahrbuch für Europarecht 2013/2014, Zürich 2014, S. 302; vgl. aber auch DAVID ROSENTHAL, Löschen und doch nicht löschen, digma 2019, S.190 ff.; TEITLER (Fn.40), S. 81 ff.; Richtlinie 7.5 der Richtlinien zur Erklärung der Pflichten und Rechte der Journalistinnen und Journalisten; Art.17 der Verordnung (EU) 2016/679 des Europäischen Parlaments und des Rates vom 27. April 2016 zum Schutz natürlicher Personen bei der Verarbeitung personenbezogener Daten, zum freien Datenverkehr und zur Aufhebung der Richtlinie 95/46/EG (Datenschutz-Grundverordnung, DSGVO) ABlL119 vom 4. Mai 2016, S.1.

43 Bundesgesetz vom 19.Juni 1992 über den Datenschutz(DSG; SR 235.1).

44 Urteil des EGMR 60798/10 und 65599/10 vom 28.Juni 2019(M.L. und W.W. gegen Deutschland), \$105.

45 HEINRICH/WEBER (Fn. 42), S. 303. 
identifizierende Berichterstattung und digitale Datenbanken langfristig andauern können. Doch kann es deswegen als achtenswerter Grund für eine Namensänderung gelten? Dazu muss zuerst geklärt werden, ob die nachteiligen Auswirkungen der Publizität rechtlich nicht sogar gewollt sind.

Während der Zweck der Anordnung einer strafrechtlichen Sanktion umstritten ist und dazu zahlreiche Theorien diskutiert werden, ${ }^{46}$ besteht beim Ziel des Straf- und Massnahmenvollzugs indes mehr Einigkeit: Dieser liegt sowohl bei bedingten, als auch bei unbedingten Strafen und bei den Massnahmen in der Resozialisierung, resp. der positiven Spezialprävention. ${ }^{47}$ Der Vollzug soll also die Fähigkeiten vermitteln - resp. die Möglichkeiten dazu nicht entziehen - deliktfrei leben zu können, während generalpräventive sowie vergeltende Überlegungen in den Hintergrund treten..$^{48}$ Dieser Zweck wird indes durch die oben beschriebenen Effekte des sozialen Stigmas potenziell gefährdet.

Weiter muss - wie von einigen der befragten Behörden vorgebracht - geklärt werden, ob eine Namensänderung überhaupt ein geeignetes Mittel darstellt, um diesem Stigma zu entgehen. Durch das Strafregister bleibt weiter ersichtlich, welche Straftaten durch eine Person begangen wurden (siehe unten IV.2) und Behörden sowie potenzielle Arbeitgebende können weiterhin darüber in Kenntnis gesetzt werden. Dennoch könnte im alltäglichen Umgang mit Dritten das Stigma des Kriminellen abgelegt werden, insbesondere auch dadurch, als dass diese nicht mehr durch eine einfache Datenbankabfrage Informationen über die kriminelle Vergangenheit erhalten könnten. Dieses partielle «dem Stigma entgehen» reicht u.E. aus, um die Namensänderung als geeignet zu erachten.

23 Zur Feststellung, ob ein achtenswerter Grund vorliegt, muss nun differenziert werden, da eine Stigmatisierung unterschiedliche Qualitäten annehmen kann. Zu berücksichtigen sind hierbei sicherlich die direkten Folgen einer Stigmatisierung, etwa Drohungen und tätliche Angriffe oder mit vergangener Straffälligkeit begründete

46 Siehe für eine Übersicht: ANNA CONINX, Rechtsphilosophische Grundlagen des Strafens und aktuelle Entwicklungen im Massnahmenrecht, recht 2016, S. $157 \mathrm{ff}$.

47 Urteil des Bundesgerichts 6B_1028/2014 vom 17.Juli 2015 E.3.2; BENJAMIN F. BRÄGGER, in: Niggli/Wiprächtiger (Hrsg.), Strafrecht (StGB/JStG), Basler Kommentar, 4. Aufl., Basel 2019, Art. 75 N1 ff. (zit. BSK StGB-BEARBEITERIN); BSK StGB-DOLGE, Art. 34 N14; ANDREA BAECHTOLD / JONAS WEBER / UELI HOSTETTLER, Strafvollzug, 3. Aufl., Bern 2016, S.113, 301; GÜNTHER STRATENWERTH / FELIX BOMMER, Schweizerisches Strafrecht Allgemeiner Teil II, Strafen und Massnahmen, 3. Aufl., Bern 2020, S. 125; DANIEL JOSITSCH / GIAN EGE / CHRISTIAN SCHWARZENEGGER, Strafrecht II, Strafen und Massnahmen, 9. Aufl., Zürich 2018, S.147.

48 BSK StGB-BRÄGGER, Art. 75 N1.
Ablehnungen von Bewerbungen um Arbeitsstellen oder Wohnungen.

In vielen Fällen wird jedoch die Verknüpfung der Folgen 24 der Stigmatisierung mit ihr selbst kaum nachweisbar sein; sodann lohnt es sich, auf gewisse Kriterien als Indizien abzustellen. ${ }^{49} \mathrm{Im}$ Vordergrund stehen hierbei sicherlich die Art und Weise der Berichterstattung - wenn diese etwa unter Einbezug von Fotos oder Vollnamennennung geschieht - und die Frage deren Legalität, ${ }^{50} \mathrm{zu}-$ dem ist aber auch die Schwere des Delikts sowie die Deliktskategorie zu beachten, da damit unterschiedliche Grade der Stigmatisierung einhergehen. ${ }^{51}$

Weitere u.E. zu berücksichtigende Kriterien betreffen die 25 Auffindbarkeit der Berichterstattung, resp. weiterer online verfügbarer Informationen zur Straftat sowie ein allfälliges persistentes Interesse der Öffentlichkeit an der verurteilten Person, welches sich etwa anhand neuer Kontaktanfragen oder fortgesetzter Berichterstattung manifestiert. Auch sollten Bemerkungen in Kommentarspalten berücksichtigt werden. Ebenfalls kann eine besonders hohe Identifikationsfunktion des Namens - etwa aufgrund dessen Seltenheit - zu beachten sein, da auch diese zu einer erhöhten Stigmatisierung führen kann.

Diese Kriterien sind nun im Einzelfall sorgfältig abzu- 26 wägen und mit anderen Fällen zu vergleichen, in welchen ein achtenswerter Grund angenommen wurde. Bei den hier zu besprechenden Fällen wird die Namensänderung nicht angestrebt, weil der getragene Name generell Nachteile bringt, sondern aufgrund der spezifischen Verknüpfung mit dem Label kriminell,52 daher handelt es sich hierbei um «subjektive Unannehmlichkeiten». Es lohnt sich jedoch der Vergleich mit den objektiv nachteiligen Namen: Als Beispiele werden in Lehre und Rechtsprechung dafür Namen wie «Kliebenschädel»,

49 Zur Zulässigkeit dieser Beweisführung, siehe MARK SCHWEIZER, Beweiswürdigung und Beweismaß, Tübingen 2015, S. $378 \mathrm{ff}$.; PETER GUYAN, Art. 157 N 13, in: Spühler/Tenchio/Infanger (Hrsg.), Schweizerische Zivilprozessordnung (ZPO), Basler Kommentar, 3. Aufl., Basel 2017.

50 Vgl. FRANZ RIKLIN, Stigmatisierungsproblematik und Tätigkeit der Medien im Rahmen der Strafverfolgung und der Prozessberichtserstattung, in: Haesler (Hrsg.), Stigmatisierung durch Strafverfahren und Strafvollzug, Diessenhofen 1981, S.132.

51 Dazu kann die Forschung zu Beurteilung der Schwere von Straftaten durch die Bevölkerung beigezogen werden, welche bspw. ergeben hat, dass Sexualdelikte als gravierender eingestuft werden als Eigentumsdelikte (vgl. MARVIN E. WOLFGANG et al., The National Survey of crime severity, Washington DC 1985; THORSTEN SELLIN / MARVIN E. WOLFGANG, The Measurement of Delinquency, New York 1964).

52 Dabei gehen wir davon aus, dass das Ziel der Namensänderung im Ablegen dieses Stigmas besteht. Ist dies nicht der Fall, was etwa annehmbar wäre bei wiederholter öffentlicher Thematisierung des Urteils durch die Person selbst (vgl. dazu Urteil des Bundesgerichts 5A 440/2020 vom 5. November 2020 E. 7.1), wäre eine Namensänderung wohl rechtsmissbräuchlich. 
«Fuchsloch» für einen Verkäufer oder «Amherd» ${ }^{53}$ (was im französischen als «ah! merde» ausgesprochen wird) angegeben, welche die Tragenden dem Spott aussetzen. ${ }^{54}$

Durch die oben gemachten Ausführungen wird klar, dass die Zuschreibung des Labels kriminell zu einem Namen in vielen Fällen deutlich gravierendere Konsequenzen für die namenstragende Person hat, als ein reines «dem Spott ausgesetzt sein», weswegen die Schwelle der oben genannten Kriterien eher tief angesetzt werden sollte. Auch im Vergleich mit den subjektiven Unannehmlichkeiten ergibt sich ein Bild, welches für eine niedrige Schwelle spricht: Denn so hat das erwähnte Beispiel der Verdeckung ausländischer Herkunft ${ }^{55}$ wohl ebenfalls das Ziel, dem mit einem Migrationshintergrund verbundenen Stigma zu entgehen, ${ }^{56}$ ohne dass dabei weitere Unannehmlichkeiten nachgewiesen werden müssten.

\section{Verhinderung des Missbrauchs}

Wie eingangs bemerkt, darf eine Namensänderung «nicht rechtswidrig, missbräuchlich oder sittenwidrig» ${ }^{57}$ sein. Insbesondere die Verhinderung eines Missbrauchs erscheint im Zusammenhang mit strafrechtlich Verurteilten besonders diskussionswürdig, zumal mehrere Kantone eine Namensänderung aufgrund dessen ablehnten. Der Begriff der Missbräuchlichkeit der Namensänderung wurde von Lehre und Rechtsprechung bis anhin nicht konkretisiert, ${ }^{58}$ weswegen es sinnvoll erscheint, Art. 2 Abs. 2 ZGB beizuziehen. Dabei ist insb. das Verbot der zweckwidrigen Verwendung von Rechtsinstituten relevant, wonach ein Missbrauch bejaht wird, «wenn das betreffende Institut zu Zwecken missbraucht wird, die offenkundig und zweifelsfrei nichts mehr gemein haben mit der Grundidee und den Zwecken, welche mit einem solchen Institut vernünftigerweise verfolgt werden».59

29 Im Zusammenhang mit der Missbräuchlichkeit hoben die Kantone zur Begründung vorrangig hervor, dass eine Identitätsverschleierung verhindert werden solle, das Informationsinteresse der Öffentlichkeit gewahrt werden müsse und aus dem Strafregister erkennbar bleiben solle, dass eine Person straffällig geworden sei.

53 Hiermit soll keinesfalls eine Anspielung auf eine amtierende Bundesrätin gemacht werden, sondern lediglich die eher tiefe Schwelle bei objektiv nachteiligen Namen verdeutlicht werden.

54 BSK ZGB I-BÜHLER, Art. $30 \mathrm{~N} 8$.

55 Vgl. oben II.

56 Vgl. dazu auch GOFFMANN (Fn. 23), S. 4 .

57 Siehe Fn. 16

58 Siehe Fn.16; CORA GRAF-GAISER, Das neue Namens- und Bürgerrecht, FamPra.ch 2013, S. 283.

59 HEINZ HAUSHEER / REGINA E. AEBI-MÜLLER, Berner Kommentar Art. 1-9 ZGB, Bern 2012, Art. 2 N 255; vgl. auch BSK-ZGB I-HONSELL, Art. 2 N 51.
Hierbei ist nun zuerst zu ergründen, welches Informa- 30 tionsinteresse der Öffentlichkeit über ehemalige Straftäterinnen und Straftäter denn überhaupt besteht: Die Diskussion hierzu wurde vor allem bzgl. der Berichterstattung über vergangene Straftaten geführt; dabei herrscht Konsens bezüglich der Tatsache, dass eine Bestrafung nach Urteilsverkündung und Ablauf einer gewissen Frist zu einem Geheimnis i.S.v. Art. 320 StGB werden kann, ${ }^{60}$ ebenso auch, dass die verbüsste Strafe der Privatsphäre zuzuordnen ist. ${ }^{61}$ Ob ein Eingriff in die Privatsphäre durch mediale Berichterstattungen in diesen Fällen gerechtfertigt werden kann, ist jedoch umstritten; die Doktrin erachtet dies generell als unzulässig, ${ }^{62}$ während das Bundesgericht eine Interessenabwägung vornimmt und dabei die Resozialisierung gegenüber dem Informationsauftrag der Medien und dem Interesse an der Straftäterin resp. dem Straftäter gewichtet. ${ }^{63}$

Durch eine Namensänderung wird der Informationsauf- 31 trag bzgl. der Straftat an sich jedoch nicht berührt, da über diese unter Verwendung des alten Namens der verurteilten Person - falls nach der etablierten Rechtsprechung zulässig - weiterhin berichtet werden kann. Auf jeden Fall liegt aber kein Missbrauch des Instituts der Namensänderung vor, liegt dessen Zweck in den zu besprechenden Fällen ja im Entgehen einer Stigmatisierung - die Verhinderung einer Berichterstattung unter Verwendung des neuen Namens steht dem nicht offenkundig entgegen, sondern ist vielmehr eine Grundvoraussetzung dafür.

Ferner stehen allfälligen Opfern der begangenen Straf- 32 taten zwar verschiedene Informationsrechte bzgl. des Vollzugsverlaufs zu. ${ }^{64}$ Diese Rechte werden jedoch durch eine Namensänderung nicht tangiert, da sie personenund nicht namensgebunden sind. Zu beachten ist ferner, dass Informationen hinsichtlich einer Namensänderung dadurch nicht erlangt werden können, da diese keinen Entscheid des Straf- und Massnahmenvollzugs darstellen. ${ }^{65}$

60 BGE127IV122 E.3a/bb S.129ff.; ULRICH WEDER, in: Donatsch (Hrsg.), StGB/JStG Kommentar, 20. Aufl., Zürich 2018, Art.366 N 3.

61 BGE122 III 449 E.3af. S. 456 f.; TEITLER (Fn. 40), s. 82 ff., m.w.H; was durch die Qualifikation als besonders schützenswerte Personendaten noch verdeutlicht wird, vgl. Art. 365 Abs. 1 StGB, Art. 3 lit. c Ziff. 4 DSG.

62 Vgl. TEITLER (Fn. 40), S. 96; TANJA IVANOVIC, Berichterstattung über aus dem Strafregister entfernte Urteile, in: Staub (Hrsg.), Beiträge zu aktuellen Themen an der Schnittstelle zwischen Recht und Betriebswirtschaft V, Zürich 2019, S. 198; FRANZ RIKLIN, Schweizerisches Presserecht, Bern 1996, \$7 N18.

63 BGE122 III 449 E. 3a ff. S. 456f.; jedoch offen gelassen in Urteil des Bundesgerichts 5A 440/2020 vom 5. November 2020 E. 6.4.1ff., etwas widersprüchlich dazu auch BGE135IV 87 E.2.4 S.92.

64 Art. 92a Abs.1 StGB.

65 Bericht der Kommission für Rechtsfragen des Nationalrats vom 7. November 2013 zur Parlamentarischen Initiative «Schaffung 

vergangene Straffälligkeit: Zahlreiche staatliche Stellen können durch das Strafregister Einblick in vergangene Straffälligkeit von Personen nehmen, ebenso ermöglicht die Einsicht in einen Privat- oder Sonderprivatauszug Dritten, Kenntnis der dort eingetragenen Urteile zu erhalten. ${ }^{66}$ Hierbei ist jedoch hervorzuheben, dass Privatpersonen nur über sich selbst Strafregisterauszüge anfordern und damit auch die Informationskundgabe deren Inhalts selbst steuern können. ${ }^{67}$ che Zwecke verwendet: Seien es Sicherheitsprüfungen, Prognosebeurteilungen oder die Information über Tätigkeitsverbote. ${ }^{68}$ Aber auch die Möglichkeit der Ausübung privatrechtlicher Tätigkeiten wird teilweise vom Inhalt des Strafregisterauszugs abhängig gemacht. ${ }^{69}$ Könnte all dies durch eine Namensänderung verhindert werden, ist u.E. eine Missbräuchlichkeit zumindest diskutabel. ${ }^{70}$

Demnach gilt zu ergründen, ob eine Namensänderung im Strafregister nachgeführt werden kann. Im Strafregister werden auch ehemalige Namen aufgeführt ${ }^{71}$ und die für den Betrieb des Strafregisters zuständige Behörde kann auch auf die Zivilstandsregister zugreifen, ${ }^{72}$ in welchem ehemalige Namen eingetragen sind ${ }^{73}$ sowie direkt Auskünfte von Zivilstandsämtern erlangen. ${ }^{74}$ Dennoch stellen sich hierbei Probleme: Da keine Bekanntgabe der Namensänderungen an die für das Strafregister zuständige Behörde von Amtes wegen erfolgt, ${ }^{75}$ müsste die Behörde die Zivilstandsregister selbst durchsuchen, resp. die Zivilstandsämter einzeln periodisch anfragen, was zu unnötigem Verwaltungsaufwand führen würde

wichtigter [sic!] Informationsrechte des Opfers» (BBl 2014 889), S. 895 e contrario. Anzumerken ist, dass der geänderte Name bei Bekanntgabe anderer Informationen u.U. mitangegeben werden kann, was wohl dem Zweck der angestrebten Namensänderung zuwiderlaufen würde und daher vermieden werden sollte. Eine abschliessende Diskussion der Problematik würde jedoch den Rahmen dieses Aufsatzes sprengen.

66 BSK StGB-ARNOLD/GRUBER, Art. 371 N 4 ff., Art. 371a N 5.

67 Auch wenn selbstverständlich aufgrund gewisser Subordinationsverhältnisse die Freiwilligkeit der Kundgabe stark eingeschränkt werden kann, vgl. BSK StGB-ARNOLD/GRUBER, Art. 371 N 8, Art. 371a N5.

68 BSK StGB-ARNOLD/GRUBER, Art. 365 N 19, Art. 369 N 7, Art. 371 a N 5.

69 BSK StGB-ARNOLD/GRUBER, Art. $371 \mathrm{~N} 7$.

70 Vgl. dazu auch Nr. 30 Abs. 4 NamÄndVw, welche bei Eintrag in das Strafregister die Weiterführung der Identifikation erfordert.

71 Anhang1,Ziff. 1.3. VOSTRA-V (Verordnung vom 29. September 2006 über das Strafregister [VOSTRA-Verordnung, VOSTRA-V; SR 331]).

72 Art. 43a Abs. 4 Ziff.3 ZGB.

73 Art. 98 Abs. 1 lit. f und g ZStV (Zivilstandsverordnung vom 28. April 2004 [ZStV; SR 211.112.2]).

74 Art.19 VOSTRA-V.

75 Art. 43a Abs. 3 ZGB i.V.m. Art. 48aff. ZStV e contrario, vgl. auch Art.19 VOSTRA-V, welcher lediglich eine Auskunftspflicht, aber kein Melderecht statuiert (zur Abgrenzung siehe Botschaft vom 20.Juni 2014 zum Strafregistergesetz [BBl 2014 5713], S. 5743f.). und die Akkuratheit des Strafregisters nicht durchgehend gewährleisten würde, womit dessen Sicherheitsfunktionen beeinträchtigt werden könnten.

Fraglich ist somit, ob eine aktive Information der für das 36 Strafregister zuständigen Behörde über die Namensänderung zulässig wäre. Da es sich dabei um eine Bearbeitung von Personendaten handeln würde, ${ }^{76}$ wäre eine gesetzliche Grundlage im materiellen Sinne oder eine Einwilligung notwendig. ${ }^{77}$ Eine solches Gesetz, welches eine Mitteilung ermöglichen würde, könnte von Bundesrechts wegen nach Art. 56 Abs. $1 \mathrm{ZStV}^{78}$ geschaffen werden. Das Verlangen einer Einwilligung zur Datenweitergabe erscheint jedoch ebenso möglich. Falls diese verweigert und so eine Nachführung des Strafregisters behindert würde, erscheint die Annahme einer Missbräuchlichkeit als zulässig, ${ }^{79}$ hat doch die Verhinderung der vorgesehenen Sicherheitsfunktionen des Strafregisters wenig gemein mit dem beabsichtigten Entgehen der Stigmatisierung.

Es stehen also bereits heute Möglichkeiten zur Nachfüh- 37 rung der Namensänderung im Strafregister zur Verfügung. Da dies der gesetzlich vorgesehene Vorgang zur Information bei sicherheitsrelevanten Tätigkeiten ist, können allfällige Sicherheitsbedenken ebenfalls beseitigt werden.

Eine allfällige Missbräuchlichkeit kann somit durch Nach- 38 führung des Strafregisters unterbunden werden, weswegen sie der Namensänderung nicht im Wege stehen sollte. ${ }^{80}$ Es ist jedoch auch anzumerken, dass sich diese Problematik in jedem Fall lediglich bis zum Inkrafttreten des neuen Strafregistergesetzes stellt, ${ }^{81}$ da in jenem durch Aufnahme der AHV13-Nummer die Identifikation einer Person und die Rückverfolgbarkeit von Namensänderungen problemlos gewährleistet sein wird. 82

76 BEAT RUDIN, in: Baeriswyl/Pärli (Hrsg.), Datenschutzgesetz (DSG), Stämpflis Handkommentar, Bern 2015, Art. 2 N18, Art. 3 N 32 ff.; BEAT RUDIN, in: Baeriswyl/Rudin (Hrsg.), Praxiskommentar zum Informations- und Datenschutzgesetz des Kantons Zürich (IDG), Zürich 2012, §3 N22 ff.

77 Art. 4Abs.1,5DSG; stellvertretend für die kantonalen Datenschutzgesetze $\$ 16$ Abs. 1 IDG/ZH (Gesetz des Kantons Zürich vom 12. Februar 2007 über die Information und den Datenschutz [IDG; ON170.4]).

78 Zivilstandsverordnung vom 28. April 2004 (ZStV; SR 211.112.2).

79 Drei Kantone gaben uns an, dass sie dies bereits heute so handhaben.

80 Selbstverständlich können auch allfällige Problematiken mit dem Betreibungsregister auftreten, welche sich jedoch ähnlich lösen lassen sollten. Zudem ermöglicht bereits ein Wohnsitzwechsel die Erlangung eines leeren Betreibungsregisterauszuges und erschwert damit die Auffindbarkeit (vgl. Art. 8 a Abs. 1 i.V.m. Art. 2 Abs.1 SchKG [Bundesgesetz über Schuldbetreibung und Konkurs vom 11. April 1889, SchKG; SR 281.1]).

81 Was voraussichtlich 2023 der Fall sein wird, siehe Revision des Strafregisterrechts.

82 Vgl. Art. 17 Abs. 1 lit. a StReG (Bundesgesetz über das Strafregister-Informationssystem VOSTRA [Strafregistergesetz, StReG]); Botschaft vom 20. Juni 2014 zum Strafregistergesetz (BBl 2014 5713), S. 5724. 


\section{Zeitpunkt}

39 änderung zuständigen Behörden ein Gesuch abgelehnt, welches sehr früh im Haftverlauf gestellt wurde; dies mit der Begründung, dass zu diesem Zeitpunkt die Resozialisierung nicht im Zentrum stünde. Es stellt sich somit die Frage, zu welchem Zeitpunkt die Namensänderung gewährt werden sollte. ständigen Behörde entnehmen wir, dass sie umso weniger von der Notwendigkeit der Namensänderung zu Resozialisierungszwecken auszugehen scheinen, je weiter entfernt die (bedingte) Entlassung der betroffenen Person ist. Implizit heisst dies nun, dass nach Behördenansicht erst nach Entstehung von Berührungspunkten mit der Gesellschaft ausserhalb der Straf- oder Massnahmenvollzugseinrichtung eine Namensänderung einer eingewiesenen Person bewilligt werden kann.

41 Die Umsetzung des Resozialisierungsziels erfolgt im schweizerischen Straf- und Massnahmenvollzug u.a. durch die Gewährung von Vollzugsprogressionen resp. Vollzugsstufen. Das Progressivsystem sieht die systematische und stufenweise Lockerung des Freiheitsentzuges vor, welche sich in der Art einer Verlegung in eine offene Anstalt, der Gewährung von Urlaub, die Zulassung zum Wohn- und Arbeitsexternat sowie der bedingten Entlassung gestalten können. ${ }^{83}$ ten oder unbegleiteten Ausgängen oder Urlauben wesentliche Berührungspunkte mit der Gesellschaft anzunehmen sind, kann durchaus diskutiert werden. Im frühestens nach Verbüssung der Hälfte der Strafe möglichen Arbeitsexternat arbeitet die eingewiesene Person ausserhalb der Straf- oder Massnahmenvollzugseinrichtung, verbringt jedoch die Ruhe- und Freizeit in der Anstalt. ${ }^{84}$ Wenn sich die inhaftierte Person im Arbeitsexternat bewährt, so erfolgt u.U. der weitere Vollzug in Form des Wohn- und Arbeitsexternats, dabei wohnt und arbeitet die inhaftierte Person ausserhalb der Anstalt, untersteht aber weiterhin der Strafvollzugsbehörde. ${ }^{85}$ Dass im Rahmen dieser Vollzugsstufen extensive Kontakte mit Personen ausserhalb der Anstalt entstehen, erscheint offensichtlich.

Die oben genannten Auswirkungen der Stigmatisierung entfalten zweifellos bei Kontakten mit der breiteren Bevölkerung ihre Wirkung, weswegen die Bewilligung der

83 BAECHTOLD/WEBER/HOSTETTLER (Fn. 47), S.164.

84 BAECHTOLD/WEBER/HOSTETTLER (Fn. 47), S. 132.

85 Vgl. Art. 77a StGB; BSK StGB-BRÄGGER, Art. 77a N1c, 1h, 12.
Namensänderung - folgt man der Namensänderungspraxis der Behörden - spätestens mit der Anordnung des Arbeitsexternats zu erteilen ist.

Die hiervor beschriebene und zur Ablehnung der Gesu- 44 che beigezogene Argumentation greift jedoch insofern zu kurz, als dass sie verkennt, dass die Verhinderung der Stigmatisierung auch umfassen kann, die betroffene Person durch die Bewilligung der Namensänderung vor der Gefährdung des Resozialisierungsprozesses und den unangenehmen Folgen des Stigmas durch Mitinsassinnen oder Mitinsassen zu bewahren. Man stelle sich bspw. die Situation eines namentlich bekannten Sexualstraftäters vor: Sollte dieser nach einer Namensänderung die Haftanstalt wechseln, könnten Mitinhaftierte nicht mit einer einfachen Suchmaschinenabfrage nachvollziehen, was ihm vorgeworfen wurde, und die Gefahr einer weiteren Stigmatisierung aufgrund der (auch) in der Gefängnispopulation besonders verpönten Taten könnte damit verhindert werden.

Deswegen plädieren wir dafür, die Gewährung der Na- 45 mensänderung nicht von durchlaufenen Progressionsstufen im Rahmen des Straf- oder Massnahmenvollzugs abhängig zu machen, sondern sie ab dem Zeitpunkt zu gewähren, ab welchem sich die Effekte der Stigmatisierung auswirken, womit auch der Perspektive von zu einer bedingten Strafe oder eine Geldstrafe verurteilten Personen Rechnung getragen werden kann.

\section{Fazit}

Wie aufgezeigt, kann eine identifizierende Medienbe- 46 richterstattung über eine strafrechtlich verurteilte Person zu zahlreichen negativen Konsequenzen für das Leben in der Gesellschaft führen. Je nach Ausprägung der Stigmatisierung sollte der Wunsch des Entgehens dessen ausreichen, um eine Namensänderung zu erlauben, wobei die Schwelle nicht zu hoch angesetzt werden darf.

Zu beachten ist dabei, dass zur Verhinderung einer Miss- 47 bräuchlichkeit das Strafregister allenfalls mit dem neuen Namen nachzuführen ist. Dies kann durch das Schaffen gesetzlicher Grundlagen oder das Einholen von Einwilligungen zur Benachrichtigung der für das Strafregister zuständigen Behörde einfach vollzogen werden. Die Namensänderung ehemaliger Straftäterinnen und Straftätern sollte bereits ab demjenigen Zeitpunkt gewährt werden, ab welchem sich die Stigmatisierung auswirkt, dieser Zeitpunkt kann auch bereits in den Vollzug einer Inhaftierung fallen.

Vor dem Hintergrund dieser Ausführungen erscheint 48 die momentane kantonale Praxis zu streng, da diese die 
negativen Auswirkungen einer Stigmatisierung als kriminell zu verkennen scheint und die Nachvollziehbarkeit einer kriminellen Vergangenheit durch die Öffentlichkeit in einer durch die rechtlichen Grundlagen nicht gestützten Weise vertritt.

BENJAMIN STÜCKELBERGER / EVIN YESILÖZ / DAMIAN CAVALLARO, Namensänderungen strafrechtlich Verurteilter

nach identifizierender Medienberichterstattung, sui generis 2021, S. 93 